\title{
High-redshift blazar identification for Swift J1656.3-3302 ${ }^{\star}$
}

\author{
N. Masetti ${ }^{1}$, E. Mason ${ }^{2}$, R. Landi ${ }^{1}$, P. Giommi ${ }^{3}$, L. Bassani ${ }^{1}$, A. Malizia ${ }^{1}$, A. J. Bird ${ }^{4}$, A. Bazzano ${ }^{5}$, A. J. Dean ${ }^{4}$, \\ N. Gehrels ${ }^{6}$, E. Palazzi ${ }^{1}$, and P. Ubertini ${ }^{5}$
}

1 INAF - Istituto di Astrofisica Spaziale e Fisica Cosmica di Bologna, via Gobetti 101, 40129 Bologna, Italy e-mail: masetti@iasfbo.inaf.it

2 European Southern Observatory, Alonso de Cordova 3107, Vitacura, Santiago, Chile

ASI Science Data Center, via Galileo Galilei, 00044 Frascati, Italy

4 School of Physics \& Astronomy, University of Southampton, Southampton, Hampshire SO17 1BJ, UK

5 INAF - Istituto di Astrofisica Spaziale e Fisica Cosmica di Roma, via Fosso del Cavaliere 100, 00133 Roma, Italy

6 NASA/Goddard Space Flight Center, Greenbelt, MD 20771, USA

Received 23 October 2007 / Accepted 14 January 2008

\section{ABSTRACT}

\begin{abstract}
We report on the high-redshift blazar identification of a new gamma-ray source, Swift J1656.3-3302, detected with the BAT imager onboard the Swift satellite and the IBIS instrument on the INTEGRAL satellite. Follow-up optical spectroscopy has allowed us to identify the counterpart as an $R \sim 19$ mag source that shows broad Lyman- $\alpha$, Si IV, He II, C IV, and C III] emission lines at redshift $z=$ $2.40 \pm 0.01$. Spectral evolution is observed in X-rays when the INTEGRAL/IBIS data are compared to the Swift/BAT results, with the spectrum steepening when the source gets fainter. The 0.7-200 keV X-ray continuum, observed with Swift/XRT and INTEGRAL/IBIS, shows the power law shape typical of radio loud (broad emission line) active galactic nuclei (with a photon index $\Gamma \sim 1.6$ ) and a hint of spectral curvature below $\sim 2 \mathrm{keV}$, possibly due to intrinsic absorption $\left(N_{\mathrm{H}} \sim 7 \times 10^{22} \mathrm{~cm}^{-2}\right)$ local to the source. Alternatively, a slope change $(\Delta \Gamma \sim 1)$ around $2.7 \mathrm{keV}$ can describe the X-ray spectrum equally well. At this redshift, the observed 20-100 keV luminosity of the source is $\sim 10^{48} \mathrm{erg} \mathrm{s}^{-1}$ (assuming isotropic emission), making Swift J1656.3-3302 one of the most X-ray luminous blazars. This source is yet another example of a distant gamma-ray loud quasar discovered above $20 \mathrm{keV}$. It is also the farthest object, among the previously unidentified INTEGRAL sources, whose nature has been determined a posteriori through optical spectroscopy.
\end{abstract}

Key words. quasars: emission lines - quasars: individual: J1656.3-3302 - galaxies: high-redshift - galaxies: active X-rays: galaxies - astrometry

\section{Introduction}

Blazars are distant and powerful active galactic nuclei (AGNs) which are oriented in such a way that a jet expelled from the central black hole is directed at small angles with respect to the observer's line of sight (for a recent review, see Padovani 2007). In the widely adopted scenario of blazars, a single population of high-energy electrons in a relativistic jet radiates over the entire electromagnetic spectrum via synchrotron and inverse Compton processes, the former dominating at low energies, the latter being relevant at high energies (Ghisellini et al. 1998). The ambient photons that are inverse Compton scattered can be either internal (synchrotron self-Compton) and/or external (external Compton scattering) to the jet. As a consequence, the spectral energy distribution (SED) of blazars shows a double-humped shape, with the synchrotron component peaking anywhere from infrared to X-rays and the inverse Compton emission extending up to $\mathrm{GeV} / \mathrm{TeV}$ gamma rays.

To explain the various SED shapes observed in blazars, Fossati et al. (1998) proposed the so-called "blazar sequence", according to which a relation between peak energies and $\gamma$-dominance (the luminosity ratio of the second to the first

\footnotetext{
* Partly based on X-ray observations with INTEGRAL, an ESA project with instruments and science data centre funded by ESA member states (especially the PI countries: Denmark, France, Germany, Italy, Switzerland, Spain), Czech Republic and Poland, and with the participation of Russia and the USA, and on optical observations collected at ESO (La Silla, Chile) under programme 079.A-0171(A).
}

peak) is present as a function of the source total power. This means that more luminous sources have both synchrotron and inverse Compton peaks located at lower energies and are more gamma-ray dominated than their fainter (and generally lower redshift) analogues.

Within the blazar population, high-redshift objects are the most luminous and generally belong to the class of Flat-Spectrum Radio Quasars (FSRQ). Observations of highluminosity blazars in the X-/gamma-ray band are particularly important (especially if available over a broad energy range) as they allow the characterization of the inverse Compton peak and related parameters. More specifically, a flattening in the spectral distribution of the seed photons producing X-rays via inverse Compton is often observed at low energies in the X-ray spectra of these objects and can be measured only with broad band data (see e.g. Tavecchio et al. 2007, and references therein).

Unfortunately, the situation is far more complex, as absorption intrinsic to the source can also reproduce the spectral curvature observed in the X-ray band (e.g., Page et al. 2005; Yuan et al. 2006); in this case, information on the absorption is useful to understand the source environment and its relation to the jet. Besides this, X-/gamma-ray observations can provide evidence for the existence of extreme blazars, i.e. those with the synchrotron peak lying at X-ray energies (Giommi et al. 2007; Bassani et al. 2007).

Here, we report detailed information on a new, powerful and hard X-ray selected blazar, Swift J1656.3-3302, recently discovered through high-energy observations made with Swift/BAT 
and INTEGRAL/IBIS. We present the results of our optical follow-up work, which has allowed the identification of the source with a blazar at redshift $z=2.4$, along with an accurate analysis of the available Swift/XRT and INTEGRAL/IBIS data. We also construct a SED for Swift J1656.3-3302 and discuss the characteristics of the source broad band emission.

The paper is structured as follows: Sect. 2 reports a collection of the main results available in the literature on this source; Sects. 3 and 4 illustrate the optical and high-energy observations, respectively; Sect. 5 contains the results of this observational campaign, while a discussion on them is given in Sect. 6. Conclusions are outlined in Sect. 7. Throughout the paper, and unless otherwise specified, uncertainties are given at the $90 \%$ confidence level. We also assume a cosmology with $H_{0}=70 \mathrm{~km} \mathrm{~s}^{-1} \mathrm{Mpc}^{-1}, \Omega_{\Lambda}=0.7$ and $\Omega_{\mathrm{m}}=0.3$.

\section{Previous information on Swift J1656.3-3302}

The high-energy source Swift J1656.3-3302 was discovered with the BAT imager (Barthelmy et al. 2005) onboard the Swift satellite (Gehrels et al. 2004) during a survey performed between December 2004 and September 2005 (Okajima et al. 2006). The object was detected at coordinates RA $=16^{\mathrm{h}} 56^{\mathrm{m}} 19^{\mathrm{s}} .2$, Dec $=$ $-33^{\circ} 01^{\prime} 48^{\prime \prime}(\mathrm{J} 2000)$, which are about 6.3 from the Galactic Plane, and with a positional uncertainty of $12^{\prime}$. Okajima et al. (2006) also reported that the $14-200 \mathrm{keV}$ BAT spectrum was very hard with a photon index $\Gamma=1.3 \pm 0.3$, and with a flux of $1.0 \times 10^{-10} \mathrm{erg} \mathrm{cm}^{-2} \mathrm{~s}^{-1}$. The source flux was variable by a factor of as high as 4 .

Subsequent pointed observations (Tueller et al. 2006) with Swift/XRT (Burrows et al. 2005) performed in June 2006 located the X-ray counterpart at a position $\mathrm{RA}=16^{\mathrm{h}} 56^{\mathrm{m}} 16.56$, Dec $=$ $-33^{\circ} 02^{\prime} 09^{\prime} 3$ (J2000), with an uncertainty of $3^{\prime \prime}$. 7 . The XRT data were fitted with an absorbed power law with $\Gamma=1.4 \pm 0.3$ and $N_{\mathrm{H}}=(3.9 \pm 0.17) \times 10^{21} \mathrm{~cm}^{-2}$. By comparing this value with the Galactic absorption column density along the direction of Swift J1656.3-3302 $\left(2.2 \times 10^{21} \mathrm{~cm}^{-2}\right.$; Dickey \& Lockman 1990), Tueller et al. (2006) suggested that it may be an extragalactic object. These authors also measured an absorbed flux in the $0.3-10 \mathrm{keV}$ band of $5.6 \times 10^{-12} \mathrm{erg} \mathrm{cm}^{-2} \mathrm{~s}^{-1}$ and predicted a flux in the $14-195 \mathrm{keV}$ band of $4.0 \times 10^{-11} \mathrm{erg} \mathrm{cm}^{-2} \mathrm{~s}^{-1}$, comparable with the BAT detection of Okajima et al. (2006).

Tueller et al. (2006) detected no sources with Swift/UVOT (Roming et al. 2005) in the XRT error circle at a limiting magnitude of 20 in the ultraviolet filters. The soft X-ray position of Swift J1656.3-3302 is consistent with the radio source NVSS J165616-330211 (having a $1.4 \mathrm{GHz}$ flux density of $410.7 \pm 12.3 \mathrm{mJy}$; Condon et al. 1998) and (albeit marginally) with the faint ROSAT source 1RXS J165616.6-330150 (Voges et al. 2000). According to Tueller et al. (2006) the ROSAT data indicate that, if the two sources are the same, spectral variability may be present. A second, weak X-ray source was also found with XRT within the BAT error circle, but Tueller et al. (2006) judged it too soft and faint to be the soft X-ray counterpart of the BAT source.

Swift J1656.3-3302 was also found to be associated with an unidentified INTEGRAL source in the $3^{\text {rd }}$ IBIS survey of Bird et al. (2007), with average 20-40 keV and 40-100 keV fluxes of $9.1 \times 10^{-12} \mathrm{erg} \mathrm{cm}^{-2} \mathrm{~s}^{-1}$ and $1.4 \times 10^{-11} \mathrm{erg} \mathrm{cm}^{-2} \mathrm{~s}^{-1}$, respectively, assuming a Crab-like spectrum. According to these observations, the source lies at coordinates RA $=16^{\mathrm{h}} 56^{\mathrm{m}} 26^{\mathrm{s}} .4$, Dec $=-33^{\circ} 02^{\prime} 49^{\prime} .2(\mathrm{~J} 2000)$. The error circle is 2.5 in radius. This position is consistent with the BAT and XRT positions reported above.

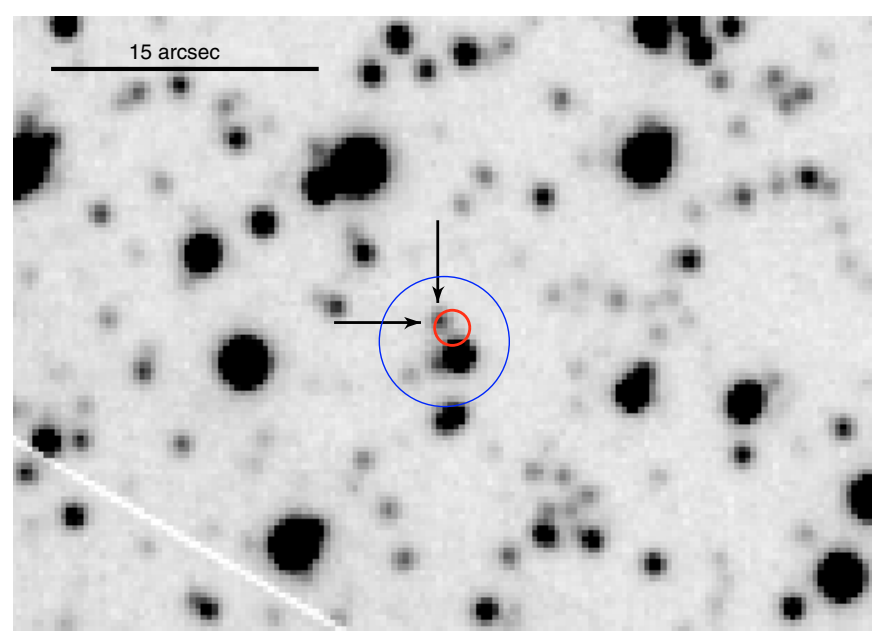

Fig. 1. Section of the ESO-3.6 m plus EFOSC2 $R$-band acquisition image (exposure time: $20 \mathrm{~s}$ ) of the field of Swift J1656.3-3302, with superimposed the X-ray $0.3-10 \mathrm{keV}$ band Swift/XRT (larger circle) and the radio $1.4 \mathrm{GHz}$ NVSS (smaller circle) positions. The actual optical counterpart, identified through optical spectroscopy at the same telescope, is indicated by the black arrows. In the image, North is at top, East is to the left.

In the following, we further analyze this source over a broad range of frequencies.

\section{Optical observations}

Medium-resolution optical spectra of the objects in the Swift/XRT error circle (see Fig. 1) were acquired between 04:24 and 06:23 UT of 21 June 2007, and between 03:34 and 04:04 UT of 24 June 2007 with the 3.6-m ESO telescope located in La Silla (Chile). This telescope carried the EFOSC2 instrument, equipped with a $2048 \times 2048$ pixel Loral/Lesser CCD. The use of grating \#13 and a slit of 1'.0 provided a 3685-9315 A nominal spectral coverage. This setup gave a dispersion of $2.8 \AA /$ pix.

The spectra (Fig. 2), after correction for cosmic-ray rejection, bias and flat-field, were optimally extracted (Horne 1986) using IRAF ${ }^{1}$. Wavelength calibration was performed using He-Ar lamps, while flux calibration was accomplished by using the spectrophotometric standard Feige 110 (Hamuy et al. 1992, 1994). The wavelength calibration uncertainty was $\sim 0.5 \AA$; this was checked by using the positions of background night sky lines.

A $20 \mathrm{~s} R$-band acquisition image of the field, secured again with the $3.6 \mathrm{~m}$ ESO telescope plus EFOSC2 under a seeing of $0 . ' 9$, was also reduced and analyzed. The image, $2 \times 2$ pixels binned, had a scale of $0.31 /$ pix and covered a field of $5.2 \times 5 ! 2$. It was corrected for bias and flat field and processed with DAOPHOT (Stetson 1987) within MIDAS ${ }^{2}$ using a PSF-fitting procedure. The choice of this photometric approach over the simple aperture photometry was dictated by the crowdedness of the field (see Fig. 1).

1 IRAF is the Image Analysis and Reduction Facility made available to the astronomical community by the National Optical Astronomy Observatories, which are operated by AURA, Inc., under contract with the U.S. National Science Foundation. It is available at http://iraf. noao. edu/

2 MIDAS (Munich Image Data Analysis System) is developed, distributed and maintained by ESO and is available at http://www. eso. org/projects/esomidas/ 


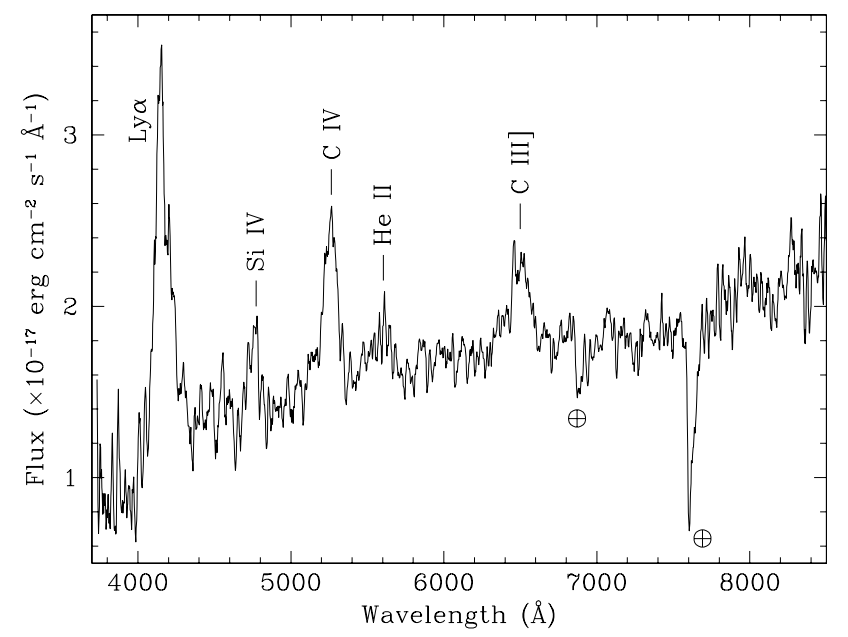

Fig. 2. 3700-8500 A optical spectrum of Swift J1656.3-3302 obtained with the ESO-3.6 m telescope. The spectrum shows broad emission lines of Lyman- $\alpha$, Si IV, He II, C IV and C III] with redshift $z=$ $2.40 \pm 0.01$. Telluric absorption bands are marked with the symbol $\oplus$. The spectrum has been smoothed with a running boxcar of 5 pixels ( 14 ̊).

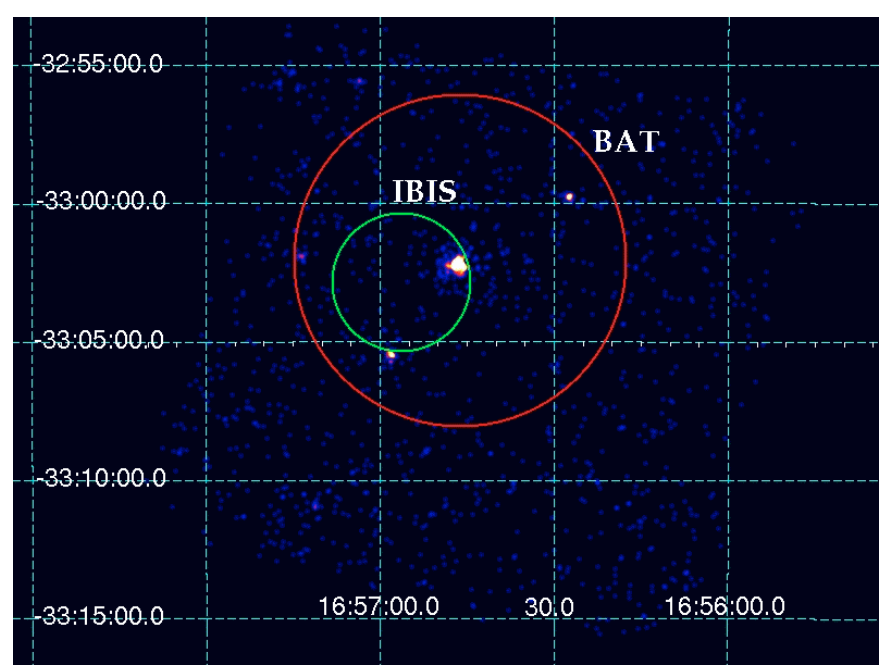

Fig. 3. Swift/XRT 0.3-10 keV image of the field of Swift J1656.3-3302 acquired on 13 June 2006. The larger circle indicates the BAT positional uncertainty and the smaller circle the IBIS one. Swift J1656.3-3302 is the brightest source in the image.

\section{X-/gamma-ray observations}

The field of Swift J1656.3-3302 was observed twice with XRT onboard Swift in the $0.3-10 \mathrm{keV}$ range (see Fig. 3). The first observation started at 03:32 UT on 9 June 2006, and the second at 16:36 UT on 13 June 2006. The pointings had on-source times of 4.4 and $4.8 \mathrm{ks}$, respectively, and both were performed in Photon Counting mode (see Burrows et al. 2005, for details on this observing mode).

Data reduction was performed using the XRTDAS v2.0.1 standard data pipeline package (XRTPIPELINE v10.0.6). Events for spectral analysis were extracted within a circular region of radius $20^{\prime \prime}$ (which encloses about $90 \%$ of the PSF at $1.5 \mathrm{keV}$; Moretti et al. 2004) centered on the source position. The background was extracted from a circular region located far from the source. In all cases, the spectra were extracted from the corresponding event files using XSELECT software and binned using GRPPHA, so that the $\chi^{2}$ statistic could reliably be used. We used version v.008 of the response matrices in the Calibration Database $^{3}$ (CALDB 2.3) maintained by HEASARC and we created individual ancillary response files using XRTMKARF v.0.5.2 within FTOOLS ${ }^{4}$ (Blackburn 1995). The X-ray spectral analysis was performed with the package XSPEC v.11.3.2 (Arnaud 1996).

We also extracted the spectral data of this source collected with the coded-mask ISGRI detector (Lebrun et al. 2003) of the IBIS instrument (Ubertini et al. 2003) onboard INTEGRAL (Winkler et al. 2003). ISGRI data were processed using the standard INTEGRAL analysis software $\left(\mathrm{OSA}^{5}\right.$ v5.1; Goldwurm et al. 2003). Events in the band 20-200 keV, coming from both fullycoded and partially-coded observations of the field of view of Swift J1656.3-3302, were included in the analysis. Details on the whole extraction procedure can be found in Bird et al. (2007). A time-averaged spectrum was obtained from the available data using the method described in Bird et al. (2006, 2007); that is, the spectra were reconstructed using the information obtained from the flux maps acquired in several bands between 20 and $200 \mathrm{keV}$. Data for a total exposure of $2.3 \mathrm{Ms}$, collected in the time interval October 2002-April 2006, were used for this task.

\section{Results}

The 0.3-10 keV image of the region around Swift J1656.3-3302 is shown in Fig. 3 with the IBIS and BAT error circles superimposed. Three objects are clearly detected within the BAT error circle. However, only one, which is also the brightest, lies within the smaller INTEGRAL error circle. This object is the soft X-ray counterpart of Swift J1656.3-3302 reported in Tueller et al. (2006).

With the data of both XRT observations we have determined its position using the most recent version of the xrtcentroid (v0.2.7) task. The correction for the misalignment between the telescope and the satellite optical axis was taken into account (see Moretti et al. 2006 for details). The position we obtained for the source is RA $=16^{\mathrm{h}} 56^{\mathrm{m}} 16^{\mathrm{s}} .83$, Dec $=-33^{\circ} 02^{\prime} 12^{\prime \prime} \cdot 3(\mathrm{~J} 2000)$, with an uncertainty of $3^{\prime \prime} 7$ on both coordinates. This is fully consistent with the preliminary results of Tueller et al. (2006).

As mentioned above, in addition to the bright X-ray source, two fainter sources are detected by XRT (see Fig. 3). One of them (the westernmost one) can readily be excluded as the soft X-ray counterpart of Swift J1656.3-3302 because it is positionally inconsistent with the IBIS error circle. The other lies 2'.62 from the center of the IBIS error circle, near its border but formally outside it. This object has coordinates RA $=16^{\mathrm{h}} 56^{\mathrm{m}} 28^{\mathrm{s}} .1$, Dec $=-33^{\circ} 05^{\prime} 25^{\prime \prime} .2(\mathrm{~J} 2000)$, with a conservative error of $6^{\prime \prime}$ on both. To further exclude any association between this source and the hard X-ray emission seen by BAT and IBIS, we examined its XRT spectrum. We found that it is very soft, as no emission is detected above $2.5 \mathrm{keV}$. Indeed, it could be fitted with a blackbody with $k T=0.5_{-0.2}^{+0.8} \mathrm{keV}\left(\chi^{2} /\right.$ d.o.f $\left.=3.8 / 3\right)$. The corresponding 2-10 keV flux is $1.3 \times 10^{-13} \mathrm{erg} \mathrm{cm}^{-2} \mathrm{~s}^{-1}$, thus (see Table 2) it is about 30 times fainter than the aforementioned bright XRT source, which is located within the INTEGRAL error circle.

The positional coincidence of this faint X-ray source with an $R \sim 11.4$ mag star in the DSS-II-red survey suggests that this soft $\mathrm{X}$-ray emission comes from a stellar corona. Indeed, mediumresolution optical spectroscopy acquired at La Silla (Chile) with

\footnotetext{
3 Available at: http://heasarc.gsfc.nasa.gov/

docs/heasarc/caldb/caldb_intro.html

${ }^{4}$ Available at: http://heasarc.gsfc.nasa.gov/ftools/

5 Available at:

http://isdc.unige.ch/index.cgi?Soft+download
} 
Table 1. Main properties of the emission lines detected in the optical spectrum of Swift J1656.3-3302. Line fluxes are reported with and without correction for the intervening Galactic extinction (see text). Equivalent Widths (EWs) are expressed in the observer's frame. Uncertainties are at $1-\sigma$ confidence level.

\begin{tabular}{|c|c|c|c|c|}
\hline \multirow[t]{2}{*}{ Line } & \multicolumn{2}{|c|}{ Flux $\left(10^{-15} \mathrm{erg} \mathrm{cm}^{-2} \mathrm{~s}^{-1}\right)$} & \multirow{2}{*}{$\begin{array}{l}E W \\
(\AA)\end{array}$} & \multirow{2}{*}{$\begin{array}{c}F W H M \\
\left(\mathrm{~km} \mathrm{~s}^{-1}\right)\end{array}$} \\
\hline & observed & $\begin{array}{l}\text { extinction- } \\
\text { corrected }\end{array}$ & & \\
\hline Lyman- $\alpha^{*}$ & $2.8 \pm 0.3$ & $39 \pm 4$ & $263 \pm 26$ & $\sim 9900$ \\
\hline Si IV & $0.41 \pm 0.12$ & $4.4 \pm 1.3$ & $30 \pm 10$ & $\sim 5000$ \\
\hline C IV & $1.19 \pm 0.12$ & $7.2 \pm 0.7$ & $80 \pm 8$ & $\sim 6600$ \\
\hline He II $^{*}$ & $0.66 \pm 0.13$ & $2.3 \pm 0.5$ & $43 \pm 9$ & $\sim 12000$ \\
\hline $\mathrm{C}$ III] $]^{*}$ & $1.1 \pm 0.1$ & $5.1 \pm 0.5$ & $65 \pm 7$ & $\sim 9300$ \\
\hline
\end{tabular}

* Possibly blended with a fainter emission line (see text).

the NTT equipped with EMMI on 2007 July 28 shows that this source is an A-type star with no peculiar spectral features.

Given all of the above multiwavelength information, we can confidently exclude the two fainter objects, detected by XRT and shown in Fig. 3, as the possible soft X-ray counterparts of Swift J1656.3-3302, and we confirm that the object reported in Tueller et al. (2006) is the soft X-ray counterpart of the source detected by BAT and IBIS.

Of the 5 optical objects within or close to the XRT error circle of Swift J1656.3-3302 (see Fig. 1), only that indicated with the tick marks in this figure shows peculiar optical spectroscopic characteristics, namely broad emission lines (Fig. 2). We identify these features as Lyman- $\alpha$, Si IV $\lambda \lambda 1394,1403, \mathrm{C}$ IV $\lambda \lambda 1548,1551$, He II $\lambda 1640$ and C III] $\lambda 1910$, at an average redshift $z=2.40 \pm 0.01$. This corresponds to a luminosity distance $d_{L}=19.4 \mathrm{Gpc}$ within the assumed cosmology. Table 1 reports the main properties of the emission lines detected in the optical spectrum of Swift J1656.3-3302.

We note that the Lyman- $\alpha$, He II and C III] emission lines have much broader Full Widths at Half Maximum (FWHMs) than the other lines. Given the resolution and the signal-to-noise ratio of the optical spectrum, this may be due to the fact that these lines may be blended with the $\mathrm{N}$ V $\lambda 1240, \mathrm{O}$ III] $\lambda 1663$ and Si III] $\lambda 1892$ emissions, respectively.

The $R$-band acquisition image was processed to obtain an astrometric solution based on several USNO-A2.0 $0^{6}$ reference stars in the field of Swift J1656.3-3302. This yields for the optical counterpart of this source the coordinates RA $=16^{\mathrm{h}} 56^{\mathrm{m}} 16^{\mathrm{s}} .853$, Dec $=-33^{\circ} 02^{\prime} 11^{\prime \prime}$.08 (J2000). The conservative error on the optical position is 0.31 , which has to be added to the systematic error of the USNO catalogue (0'.25 according to Assafin et al. $2001 \&$ Deutsch 1999). The final 1- $\sigma$ astrometric uncertainty on the optical position of Swift J1656.3-3302 is thus 0.' 40 .

This position is consistent with that of the radio source NVSS J165616-330211, indicating that the two sources are the same. This is also consistent with the position of the faint ROSAT source reported by Tueller et al. (2006). We instead exclude that the optical source USNO-A2.0 0525-24886745 (i.e. the brightest object within the XRT error circle in Fig. 1, proposed by Tueller et al. (2006), as a possible optical counterpart) is related with Swift J1656.3-3302 on the basis of its optical spectrum, which is typical of a Galactic star.

Again using USNO-A2.0 field stars as calibrators, we obtained for the true counterpart a magnitude $R=19.1 \pm 0.1$.

\footnotetext{
6 The USNO-A2.0 catalogue is available at http://archive.eso.org/skycat/servers/usnoa
}

Table 2. Best-fit parameters of the models adopted to describe the X-ray spectrum of Swift J1656.3-3302.

\begin{tabular}{lr}
\hline \hline \multicolumn{1}{c}{ Parameter } & \multicolumn{1}{c}{ Value } \\
\hline \multicolumn{1}{c}{ power law plus Galactic absorption } \\
$\chi^{2} /$ d.o.f. & $45.6 / 34$ \\
$\Gamma$ & $1.28 \pm 0.10$ \\
XRT/IBIS intercalibr. constant & $0.7_{-0.2}^{+0.4}$ \\
$F_{(2-10 \mathrm{keV})}$ & $4.9 \times 10^{-12}$ \\
$F_{(20-100 \mathrm{keV})}$ & $1.9 \times 10^{-11}$ \\
\hline \multicolumn{1}{c}{ power law plus Galactic and intrinsic absorption } \\
$\chi^{2} /$ d.o.f. & $25.2 / 33$ \\
$N_{\mathrm{H}}\left(10^{22} \mathrm{~cm}^{-2}\right)$ & $6.7_{-2.7}^{+3.2}$ \\
$\Gamma \quad$ & $1.64 \pm 0.16$ \\
$\mathrm{XRT} / \mathrm{IBIS}$ intercalibr. constant & $1.9_{-0.7}^{+1.3}$ \\
$F_{(2-10 \mathrm{keV})}$ & $4.6 \times 10^{-12}$ \\
$F_{(20-100 \mathrm{keV})}$ & $2.2 \times 10^{-11}$ \\
\hline \multicolumn{1}{c}{ broken power law plus Galactic absorption } \\
$\chi^{2} /$ d.o.f. & $23.6 / 32$ \\
$\Gamma_{1}$ & $0.86_{-0.26}^{+0.23}$ \\
$\Gamma_{2}$ & $1.81_{-0.13}^{+0.23}$ \\
$E_{\text {break }}(\mathrm{keV})$ & $2.7_{-0.5}^{+1.5}$ \\
XRT/IBIS intercalibr. constant & $2.9_{-1.3}^{+1.5}$ \\
$F_{(2-10 \mathrm{keV})}$ & $4.4 \times 10^{-12}$ \\
$F_{(20-100 \mathrm{keV})}$ & $2.0 \times 10^{-11}$ \\
\hline
\end{tabular}

Note: In the above fits, when needed, we fixed the redshift of the source at $z=2.40$ and the Galactic hydrogen column at $N_{\mathrm{H}}^{\mathrm{Gal}}=2.2 \times 10^{21} \mathrm{~cm}^{-2}$. The reported fluxes (in the observer's frame) are in $\mathrm{erg} \mathrm{cm}^{-2} \mathrm{~s}^{-1}$ and are corrected for the total intervening absorption column density. For the broken power law model, the fit parameters are computed in the observer's frame, and not in the source rest frame.

Towards the line of sight of the source, the Galactic foreground reddening is $E(B-V)=0.624$ mag (Schlegel et al. 1998). Using the law by Cardelli et al. (1989), this implies that the Galactic extinction in the $R$ band is $A_{R}=1.6$. Thus, the dereddened $R$-band magnitude of the source is $R_{0}=17.5$.

A simple power law model (as employed by Okajima et al. 2006 to characterize the BAT spectrum) provides a good description of the IBIS data but yields a steeper spectrum $(\Gamma=1.9 \pm$ 0.3 ), combined with a lower (by a factor of 3 compared to the BAT measurement) 14-195 keV flux $\left(3.4 \times 10^{-11} \mathrm{erg} \mathrm{cm}^{-2} \mathrm{~s}^{-1}\right)$. Thus, the source appears to experience spectral evolution.

We then used the Swift/XRT data to describe the X-ray spectrum of Swift J1656.3-3302, employing first a simple power law in the source rest frame (zpowerlw model in XSPEC) absorbed by the Galactic column density (reported in Sect. 2). This model provides consistent results for the two observations $(\Gamma=1.22 \pm 0.16$ for the first one and $\Gamma=1.13 \pm 0.16$ for the second). No significant flux variations are observed either during each XRT pointing or between them. Thus, to improve the statistics, we have stacked the two XRT spectra and repeated the analysis. The simple power law again provides an acceptable fit $\left(\chi^{2} /\right.$ d.o.f. $\left.=24.8 / 25\right)$ and yields a flat spectrum $(\Gamma=1.18 \pm 0.11)$.

Next, we have combined the XRT spectrum with that from IBIS, introducing a constant to allow for intercalibration differences between the two instruments; this constant was left free to vary in the fits. We are aware that, since the XRT and IBIS observations were not simultaneous, flux variations are 
plausible given the blazar nature of the object, and may also be the cause of different normalizations. Given that our past experience tells us that the intercalibration constant between these two instruments is $\sim 1$, we suggest that different values for this constant are likely to be due to the flux variability mentioned above.

Again using an absorbed power law fit with the $\mathrm{N}_{\mathrm{H}}$ value fixed to the Galactic one along the source line of sight, we obtained a best-fit photon index $\Gamma=1.28 \pm 0.10\left(\chi^{2} /\right.$ d.o.f. $=$ 45.6/34). However, this model results in fit residuals (see Fig. 4, upper panel) that show some curvature on the lower energy side, possibly due to intrinsic absorption in the source rest frame. The addition of this extra absorption component (zwabs in XSPEC) provides a fit improvement which is significant at the $95 \%$ confidence level according to the F-test (Bevington 1969). This leads to a more typical AGN spectrum $(\Gamma=1.64 \pm 0.16)$ and gives a relatively high column density $\left(N_{\mathrm{H}}=6.7_{-2.7}^{+3.2} \times 10^{22} \mathrm{~cm}^{-2}\right)$ local to the blazar (Fig. 4, central panel).

Alternatively, one may assume that the turnover in the X-ray spectrum is produced by an intrinsic slope change: in this case, by fitting the XRT and IBIS data using a broken power law absorbed by the Galactic hydrogen column, we find a spectral steepening $\Delta \Gamma=0.95$ occurring at energy $E_{\text {break }}=2.7 \mathrm{keV}$ (Fig. 4, lower panel). Again using the F-test, we find that the improvement significance of this spectral description over the simple power law with Galactic absorption is $95 \%$ in this case also.

The XRT+IBIS global fit results for the three models above are reported in Table 2. With the assumed cosmology and using the redshift of the source, we get $2-10 \mathrm{keV}$ and $20-100 \mathrm{keV}$ observer's frame luminosities of $2.1 \times 10^{47} \mathrm{erg} \mathrm{s}^{-1}$ and $9.9 \times$ $10^{47} \mathrm{erg} \mathrm{s}^{-1}$ respectively, assuming isotropic emission. These figures are largely independent of the assumed spectral model.

\section{Discussion}

Within our optical spectroscopy program aimed at the identification of the nature of sources detected at high energies with INTEGRAL (see Masetti et al. 2006, and references therein), we discovered that the object Swift J1656.3-3302 is a high-redshift blazar, located at $z=2.40$. This source is thus another case belonging to the small but growing class of distant gamma-ray emitting blazars, which are being discovered by Swift/BAT and INTEGRAL/IBIS (Sambruna et al. 2006; Sambruna et al. 2007; Bassani et al. 2007; Bird et al. 2007). Swift J1656.3-3302 is moreover the farthest object, among the previously unidentified INTEGRAL sources, whose nature has been determined a posteriori through optical spectroscopy.

The better optical position has allowed us to associate it with a fairly bright NVSS radio source and with a faint ROSAT object. The NVSS image of the source shows that it is core-dominated with no extended radio features. The optical $R$-band information reported in Sect. 4, combined with the radio flux density, suggests that $\log \left(S_{1.4 \mathrm{GHz}} / S_{R}\right) \sim 3.3$ for this object. Although this ratio is not conventionally used to characterize the radio loudness of a source, it can nevertheless be used to quantify the strength of the radio emission compared to the optical. In this estimate we did not consider any contribution from the optical extinction local to the source: to take this issue into account, we can assume for Swift J1656.3-3302 the lower extreme in the range of the optical-to-X-ray slopes typical for this kind of object (e.g., Stocke et al. 1991). This gives a strict upper limit to the $R$-band flux of the source, and hence a lower limit for the radio-optical flux density ratio: with this procedure, we obtain that $\log \left(S_{1.4 \mathrm{GHz}} / S_{R}\right)>2.5$. In summary, the considerations
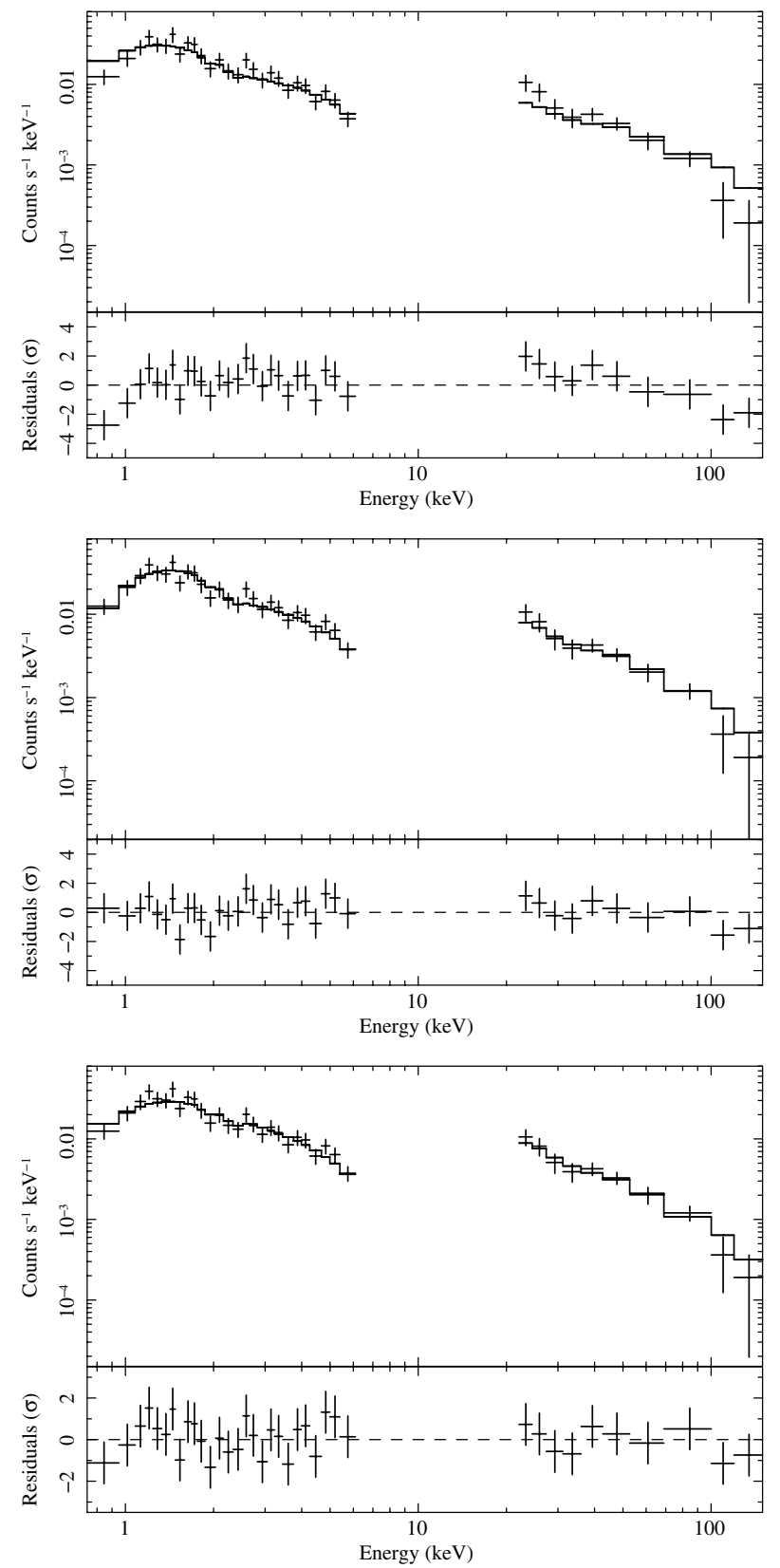

Fig. 4. Averaged 0.7-200 keV X-ray spectrum of Swift J1656.3-3302 obtained from the XRT and ISGRI data described in the text and fitted with a power law absorbed only by the Galactic hydrogen column (upper panel), a power law absorbed by Galactic plus neutral hydrogen local to the blazar (central panel), and a broken power law absorbed by the Galactic hydrogen column only (lower panel). The fit residuals using the best-fit models reported in Table 2 are shown in each panel.

above point to the fact that Swift J1656.3-3302 is very likely to be radio loud.

If we compare the source unabsorbed monochromatic X-ray flux at $1 \mathrm{keV}$ to the radio flux at $1.4 \mathrm{GHz}$, we find that their ratio is around 150 or, following the notation of Giommi et al. (2007), $\sim 2 \times 10^{-12} \mathrm{erg} \mathrm{cm}^{-2} \mathrm{~s}^{-1} \mathrm{Jy}^{-1}$, i.e. similar to blazars with the inverse Compton peak in the gamma-ray band. The source is indeed very powerful at high energies, which explains the detection by BAT and IBIS. The above properties suggest that Swift J1656.3-3302 is a distant blazar in which the emission is relativistically beamed and with a double-peaked SED. 
To verify this, we constructed the non-simultaneous SED of Swift J1656.3-3302 by combining all the available data in order to cover as many frequencies as possible (see Fig. 5). Along with the optical and X-/gamma-ray information obtained in the present work, we have used the $1.4 \mathrm{GHz}$ NVSS radio flux from Condon et al. (1998), the IRAS infrared upper limits (IRAS $1988)$ in the mid-infrared bands $(12,25,60$ and $100 \mu \mathrm{m})$, the ROSAT 0.1-2.4 keV flux (Voges et al. 2000) and the upper limit at MeV energies from the EGRET survey (Hartman et al. 1999). When plotting the SED, we respectively corrected the optical and the X-ray spectra from the contribution of the foreground Galactic extinction using the $E(B-V)$ and $N_{\mathrm{H}}$ values reported in Sects. 5 and 2, respectively. Moreover, the optical spectrum was rebinned at $\sim 60 \AA$ to smooth out the noise of the continuum, and the most evident emission lines were removed.

Looking at the SED of this object, two things are immediately clear: (i) it is indeed double peaked as expected from a blazar; and (ii) it is typical of a powerful blazar with the synchrotron peak somewhere in the infrared range and that of the inverse Compton component just above the ISGRI band (20-100 keV). Thus, given the source luminosity, the opti$\mathrm{cal} /$ radio information and the overall SED, it is likely that Swift J1656.3-3302 belongs to the class of FSRQs.

An interesting point to recall here is the fact that, as mentioned in Sect. 5, the high-energy spectrum of this source shows a deficit of soft X-ray photons. This could be produced by either an intrinsically curved shape of the spectrum itself, or absorption local to the AGN. This feature is becoming common place, rather than the exception, for high redshift blazars and it is a widely debated issue at the moment. Observations of intrinsic absorption in high redshift blazars have been reported for many objects (Page et al. 2005; Yuan et al. 2006, and references therein): the common interpretation is that this absorption originates from the material present in the AGN environment.

From the above mentioned literature there is some evidence of a correlation between absorption and redshift, with the more distant sources being more absorbed. The increase in $N_{\mathrm{H}}$ with $z$ seems to occur starting at $z \sim 2$. Moreover, at this redshift one sees a change in the fraction of radio loud quasars showing X-ray absorption, being lower at lower $z$. Finally, there is a tendency for objects with intrinsic absorption to have systematically higher X-ray fluxes. All this evidence suggests that there may be a strong cosmic evolution effect that takes place at $z \sim 2$.

Within this scenario Swift J1656.3-3302 is quite interesting, because it has a redshift larger than 2 and also because it has a relatively high column density compared to the objects discussed by Yuan et al. (2006, see their Fig. 6).

The absorption scenario is however far from being confirmed: the fact that absorption is more often seen in radio loud objects, i.e. those which more likely host a relativistic jet, raises the question of why these jets are not able to remove the gas in their vicinity. Another problem is the apparent absence of extinction at lower energies (that is, UV and optical), if absorption is indeed present in these objects, and in particular in Swift J1656.3-3302: indeed, the shape of its optical spectrum, once corrected for the Galactic extinction, suggests a local column density which is much lower than that inferred from the X-ray data analysis (this value would imply a $V$-band rest-frame extinction $A_{V} \sim 40 \mathrm{mag}$, assuming the Galactic extinction law). This effect may however be partly alleviated by the fact that, at high redshift, the chemical composition of the dust is substantially different from that of the Milky Way, thus producing an optical-UV extinction law radically at variance with that of our Galaxy (e.g., Calzetti et al. 1998; Maiolino et al. 2001).

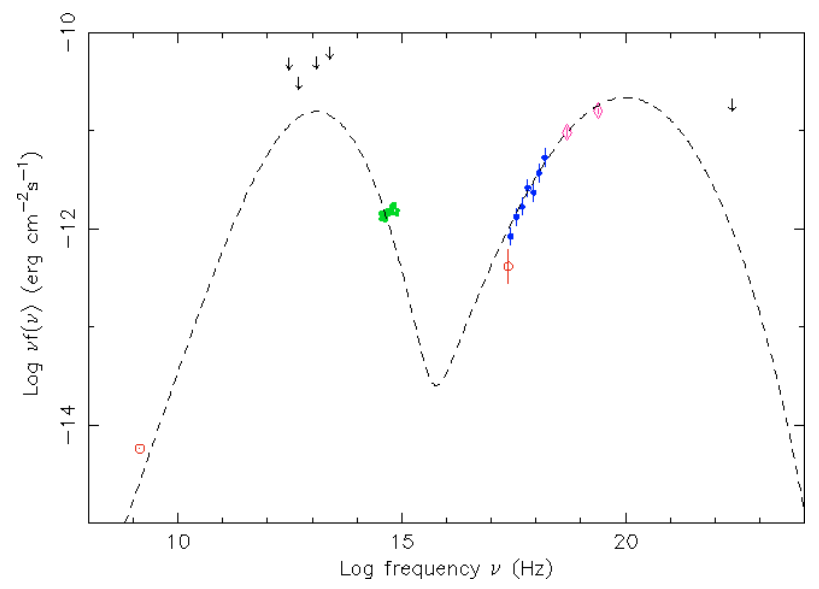

Fig. 5. Broadband non-simultaneous observational radio to gamma-ray SED of Swift J1656.3-3302, constructed with the measurements collected from the literature and with those presented in this paper (see text for details). Moving towards higher energies, the following information was used: the $1.4 \mathrm{GHz}$ NVSS radio flux, the ESO $3.6 \mathrm{~m}$ optical spectrum, the ROSAT 0.1-2.4 keV flux, and the XRT+IBIS 0.7-200 keV spectrum. The IRAS upper limits in the mid-infrared bands and the upper limit at $\mathrm{MeV}$ energies from the EGRET survey are also reported. The dashed line indicates an example of the application of the logparabolic model (Massaro et al. 2006; Tramacere et al. 2007) briefly described in the text.

An alternative interpretation for the deficit of soft photons has been put forward for blazars, i.e. that the observed shape is due to intrinsic curvature of the inverse Compton emission (Fabian et al. 2001; see also Tavecchio et al. 2007, for a recent investigation made using this model). Indeed, a low energy cutoff in the relativistic particle distribution at $\gamma_{\text {cut }}$ would produce a flattening in the scattered spectrum below $v_{\mathrm{obs}} \sim$ $v_{\mathrm{ex}} \cdot \Gamma_{\mathrm{L}}^{2} \cdot \gamma_{\text {cut }}^{2} \cdot(z+1)^{-1}$, where $\Gamma_{\mathrm{L}}$ is the Lorentz factor, $v_{\mathrm{ex}}$ the frequency of the seed photons and $z$ the redshift of the source. If $v_{\mathrm{ex}}$ is $\approx 10^{15} \mathrm{~Hz}$, the break observed in Swift J1656.3-3302 implies, for Lorentz factors in the range $10-50$, that $\gamma_{\text {cut }} \approx$ a few.

A different description of the source SED, again in the assumption of the intrinsic curvature hypothesis, can be made assuming a log-parabolic distribution for the emitting electrons, in the form $F(E)=K \cdot E^{-(a+b \cdot \log E)}$ (Massaro et al. 2006; Tramacere et al. 2007), where $a$ is the power law slope at $1 \mathrm{keV}$ and $b$ is the curvature below this energy. As an example, in Fig. 5 we overplotted on the SED a log-parabolic distribution with $a=0.55$ and $b=0.6$. We however defer the detailed analysis of the intrinsic curvature of the X-ray spectrum of Swift J1656.3-3302 to a subsequent paper.

We conclude this section by briefly commenting on the spectral evolution seen when comparing the IBIS and the BAT spectra: apparently, the source X-ray spectrum steepens when it gets fainter. The observed X-ray variability in both flux and spectrum reported here for Swift J1656.3-3302 is unusual, though not unprecedented in FSRQs. Indeed, a number of other high- $z$ blazars show the same behavior, such as RX J1028.6-0844 $(z=4.2$; Yuan et al. 2005) and GB B1428+4217 $(z=4.7$; Worsley et al. 2006) and, in more recent studies, $\operatorname{RBS} 315(z=2.7$; Tavecchio et al. 2007) and QSO 0836+710 $(z=2.2$; Sambruna et al. 2007). This variability could be due to changes in the electron distribution at low energies, or may be a hint of the presence of an extra component, which becomes stronger or weaker as the source brightness changes. 


\section{Conclusions}

Through optical follow-up observations we have been able to identify the newly discovered hard X-ray source Swift J1656.3-3302 with a blazar at $z=2.4$. Spectral evolution is observed when comparing the Swift/BAT and INTEGRAL/IBIS data, with the X-ray spectrum undergoing softening when the emission becomes fainter. The source broadband X-/gamma-ray spectrum is well described by a power law of index $\Gamma \sim 1.6$. The source is extremely bright with a $20-100 \mathrm{keV}$ observer's frame luminosity of $\sim 10^{48} \mathrm{erg} \mathrm{s}^{-1}$, assuming isotropic emission.

The source SED is typical of high luminosity blazars, as it has two peaks: the lower (synchrotron) one likely located at infrared frequencies, and the higher (inverse Compton) one positioned above a few hundred $\mathrm{keV}$. The spectral curvature detected in the X-ray spectrum of the object can either be intrinsic and due to the distribution of the emitting electrons, or associated with the presence of absorption local to the source and produced by a column density of a $\sim 7 \times 10^{22} \mathrm{~cm}^{-2}$, higher than that typically observed in high- $z$ blazars.

All of the observational evidence gathered so far therefore points to Swift J1656.3-3302 being another case of a distant luminous blazar selected at gamma-ray energies. It is also the farthest object, among the previously unidentified INTEGRAL sources, whose nature has been determined a posteriori through optical spectroscopy.

Acknowledgements. We thank A. Sánchez for night assistance at the $3.6 \mathrm{~m} \mathrm{ESO}$ telescope, A. Ederoclite for help and useful advices with the ESO telescopes, and J. B. Stephen for helpful discussions. An anonymous referee is acknowledged for comments which helped us to improve this paper. This research has made use of the NASA's Astrophysics Data System, of the HEASARC archive, and of the SIMBAD database operated at CDS, Strasbourg, France. The authors acknowledge the ASI and INAF financial support via ASI-INAF grants I/023/05/0 and $\mathrm{I} / 088 / 07 / 0$.

\section{References}

Arnaud, K. A. 1996, XSPEC: the first ten years, in Astronomical Data Analysis Software and Systems V, ed. G. H. Jacoby, \& J. Barnes, ASP Conf. Ser., 101, 17

Assafin, M., Andrei, A. H., Vieira Martins, R., et al. 2001, ApJ, 552, 380

Bassani, L., Landi, R., Malizia, A., et al. 2007, ApJ, 669, L1

Barthelmy, S., Barbier, L. M., Cummings J., et al. 2005, Space Sci. Rev., 120, 95

Bevington, P. R. 1969, Data reduction and error analysis for the physical sciences (New York: McGraw-Hill Book Company)

Bird, A. J., Barlow, E. J., Bassani, L., et al. 2006, ApJ, 636, 765
Bird, A. J., Malizia, A., Bazzano, A. et al. 2007, ApJS, 170, 175

Blackburn, J. K. 1995, FTOOLS: A FITS Data Processing and Analysis Software Package, in Astronomical Data Analysis Software and Systems IV, ed. R. A. Shaw, H. E. Payne, \& J. J. E. Hayes, ASP Conf. Ser., 77, 367

Burrows, D., Hill, J. E., Nousek, J. A., et al. 2005, Space Sci. Rev., 120, 165

Calzetti, D., Kinney, A. L., \& Storchi-Bergmann, T. 1998, ApJ, 429, 582

Cardelli, J. A., Clayton, G. C., \& Mathis, J. 1989, ApJ, 345, 245

Condon, J. J., Cotton, W. D., Greisen, E. W., et al. 1998, AJ, 115, 1693

Deutsch, E. W. 1999, AJ, 118, 1882

Dickey, J. M., \& Lockman, F. J. 1990, ARA\&A, 28, 215

Fabian, A. C., Celotti, A., Iwasawa, K., et al. 2001, MNRAS, 323, 373

Fossati, G., Maraschi, L., Celotti, A., Comastri, A., \& Ghisellini, G. 1998, MNRAS, 299, 433

Gehrels, N., Chincarini, G., Giommi, P., et al. 2004, ApJ, 611, 1005

Ghisellini, G., Celotti, A., Fossati, G., Maraschi, L., \& Comastri, A. 1998, MNRAS, 301, 451

Giommi, P., Massaro, E., Padovani, P., et al. 2007, A\&A, 468, 97

Goldwurm, A., David, P., Foschini, L., et al. 2003, A\&A, 411, L223

Hamuy, M., Walker, A. R., Suntzeff, N. B., et al. 1992, PASP, 104, 533

Hamuy, M., Suntzeff, N. B., Heathcote, S. R., et al. 1994, PASP, 106, 566

Hartman, R. C., Bertsch, D. L., Bloom, S. D., et al. 1999, ApJS, 123, 79

Horne, K. 1986, PASP, 98, 609

IRAS 1988, The Point Source Catalog, version 2.0, NASA RP-1190

Lebrun, F., Leray, J. P., Lavocat, P., et al. 2003, A\&A, 411, L141

Maiolino, R., Marconi, A., \& Oliva, E. 2001, A\&A, 365, 37

Masetti, N., Morelli, L., Palazzi, E. et al. 2006, A\&A, 459, 21

Massaro, E., Tramacere, A., Perri, M., Giommi, P., \& Tosti, G. 2006, ApJ, 448, 861

Moretti, A., Campana, S., Tagliaferri, G., et al. 2004, SPIE Proc., 5165, 232

Moretti, A., Perri, M., Capalbi, M., et al. 2006, A\&A, 448, L9

Okajima, T., Tueller, J., Markwardt, C., et al. 2006, ATel 799

Padovani, P. 2007, Ap\&SS, 309, 63

Page, K. L., Reeves, J. N., O’Brien, P. T., \& Turner, M. J. L. 2005, MNRAS, 364, 195

Roming, P. W. A., Kennedy, T. E., Mason, K. O., et al. 2005, Space Sci. Rev., 120,143

Sambruna, R. M., Markwardt, C. B., Mushotzky, R. F., et al. 2006, ApJ, 646, 23 Sambruna, R. M., Tavecchio, F., Ghisellini, G., et al. 2007, ApJ, 669, 884

Schlegel, D. J., Finkbeiner, D. P., \& Davis, M. 1998, ApJ, 500, 525

Stetson, P. B. 1987, PASP, 99, 191

Stocke, J. T., Morris, S. L., Gioia, I. M., et al. 1991, ApJS, 76, 813

Tavecchio, F., Maraschi, L., Ghisellini, G., et al. 2007, ApJ, 665, 980

Tramacere, A., Giommi, P., Massaro, E., et al. 2007, A\&A, 467, 501

Tueller, J., Markwardt, C., Ajello, M., et al. 2006, ATel, 835

Ubertini, P., Lebrun, F., Di Cocco, G., et al. 2003, A\&A, 411, L131

Voges, W., Aschenbach, B., Boller, T., et al. 2000, IAU Circ. 7432

Winkler, C., Courvoisier, T. J.-L., Di Cocco, G., et al. 2003, A\&A, 411, L1

Worsley, M. A., Fabian, A. C., Pooley, G. G., \& Chandler, C. J. 2006, MNRAS, 368,844

Yuan, W., Fabian, A. C., Celotti, A., McMahon, R. G., \& Matsuoka, M. 2005, MNRAS, 358, 432

Yuan, W., Fabian, A. C., Worsley, M. A., \& McMahon, R. G. 2006, MNRAS, 368,985 